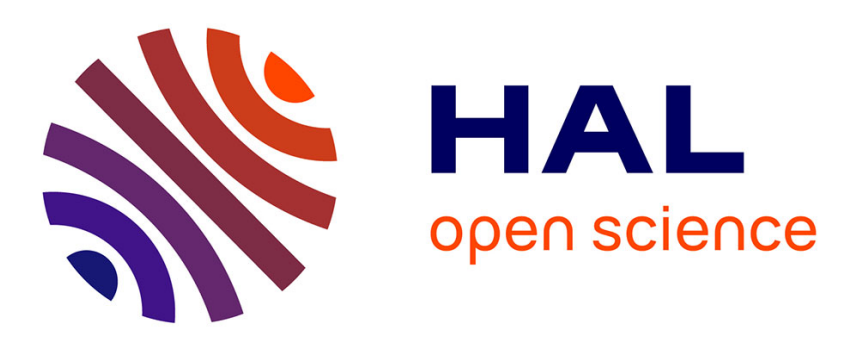

\title{
Imaging subwavelength holes in chromium films in scanning near-field optical microscopy. Comparison between experiments and calculation
}

S. Ducourtieux, S. Grésillon, J.C Rivoal, C. Vannier, C. Bainier, C. Courjon, H. Cory

\section{To cite this version:}

S. Ducourtieux, S. Grésillon, J.C Rivoal, C. Vannier, C. Bainier, et al.. Imaging subwavelength holes in chromium films in scanning near-field optical microscopy. Comparison between experiments and calculation. European Physical Journal: Applied Physics, 2004, 26 (1), pp.35-43. 10.1051/epjap:2004014 . hal-00095444

\section{HAL Id: hal-00095444 \\ https://hal.science/hal-00095444}

Submitted on 22 Apr 2021

HAL is a multi-disciplinary open access archive for the deposit and dissemination of scientific research documents, whether they are published or not. The documents may come from teaching and research institutions in France or abroad, or from public or private research centers.
L'archive ouverte pluridisciplinaire HAL, est destinée au dépôt et à la diffusion de documents scientifiques de niveau recherche, publiés ou non, émanant des établissements d'enseignement et de recherche français ou étrangers, des laboratoires publics ou privés. 


\title{
Imaging subwavelength holes in chromium films in scanning near-field optical microscopy. Comparison between experiments and calculation
}

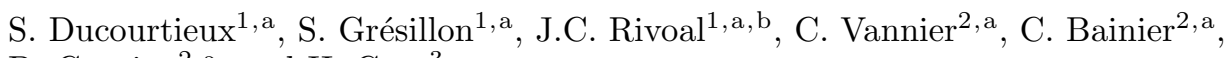 \\ D. Courjon ${ }^{2, a}$, and H. Cory ${ }^{3}$ \\ 1 CNRS UPR 5 and Univ. P. et M. Curie., Lab. d'Optique Physique, ESPCI, 10 rue Vauquelin, \\ 75231 Paris Cedex 05, France \\ 2 Laboratoire d'Optique P.M. Duffieux, CNRS UMR 6603, Université de Franche-Comté, \\ 25030 Besançon Cedex, France \\ 3 Department of Electrical Engineering, Technion - Israel Institute of Technology, Haifa 32000, Israel
}

\begin{abstract}
Near-field optical signals are imaged in the vicinity of nano-holes using two different near-field optical microscopes. The experimental results are compared with electromagnetic field calculations based on a modal approximation. It turns out that an optical fibre detects the Poynting vector whereas the apertureless tip is sensitive to the field amplitude.
\end{abstract}

Keywords. Microscopy of surfaces, interfaces and thin films, Near-field scanning optical microscopes, Atomic force microscopes

\section{Introduction}

Scanning near-field optical microscopy has undergone a remarkable growth over the last decade [1]. The various techniques which have been used in the design of near field optical microscope involve either an apertureless diffusion tip or a sharpened optical fibre as a local probe to map the optical near field over the sample surface. Advances in instrumentation correlated with theory and modelling have led to a better understanding of the signals which are detected in the near zone. A recent review of both theoretical formalism used and experimental potentialities can be found in reference [2]. However, well defined test samples investigated with various types of Scanning NearField Microscope (SNOM) or Scanning Tunnelling Optical Microscope (STOM) working in several illumination modes are sparse. The investigations on such test samples have stimulated much efforts in the CNRS framework of the French Research Group "Optique du Champ Proche". A comparison of the test images recorded by means of a set of five different microscopes has been made recently [3]. The samples investigated are truncated circular holes created in a chromium layer deposited on a cover glass slide. Such test objects are well suited to understand the nature of the optical signal recorded by different apparatus. The

\footnotetext{
a Groupement de Recherche "Optique du Champ Proche", CNRS 1860.

b e-mail: rivoal@optique.espci.fr
}

nominal diameter of the test sample is close to half the wavelength of the excitation source as it was for the subwavelength aperture studied by Lezec et al. [4]. Metal is chromium instead of silver avoiding the possibility of surface plasmon excitation when studied in the visible optical domain.

It is well known that sharpened optical fibres can detect the intensity of the evanescent field $[5,6]$. Moreover the known field enhancement at the metallic tip apex [7-10] could allow the detection of the axial component of the electromagnetic field [11]. This assumption was made in recent studies [12].

The main purpose of the present study is to correlate the near-field optical signal experimentally obtained with a crude theoretical approach. It appears in reference [3] that in similar illumination geometries, signals differ significantly depending on fibre or tip detection. We address the question: could the same theoretical model be used to interpret the images obtained with two different devices one using a fibre and the other a metallic tip? In Section 2 we describe the investigated samples. Two set-ups working in transmission mode are chosen, their characteristics are depicted in Section 3. The near-field optical images are presented in Section 4 and the formalism used to model the near-field optical signals is explained in Section 5. A comparison between experiment and computed results is made in Section 6 and in the last section conclusions are given. 


\section{Samples preparation}

\subsection{Sample preparation}

Test samples are prepared from deposition of polystyrene latex beads (diameter $\sim 300 \mathrm{~nm}$ ) onto a thin microscope plate (thickness $400 \mathrm{~nm}$ ) using a method close to that described in reference [13]. The substrate is cleaned with distilled water and ethanol, and dried in clean ambient conditions. Latex spheres in water dilution are deposited onto the surface. After drying one obtains isolated spheres or small groups of spheres or even mono-layers depending on suitable concentration of beads versus the area of deposition. A chromium layer of a few nanometers of thickness is then evaporated on the surface and finally the latex spheres are chemically lifted off. A random distribution of perfectly circular holes is obtained reproducing faithfully the original positions of the spheres before removal.

\subsection{Sample characterization by SEM or AFM imaging}

A typical SEM image is presented in Figure 1. It shows the imprint of the latex spheres in the metal surface. A group of undissolved latex spheres remaining on the surface can also be observed. Scanning the surface with an AFM tip enables to determine the radius of the isolated holes and the approximate depth of the chromium layer. This measurement does not take into account the convolution by the conical tip.

The measured average diameter of the holes is less than $200 \mathrm{~nm}$ at the bottom and about $350 \mathrm{~nm}$ at the top of the chromium surface. Their depth is about $25 \mathrm{~nm}$ as shown in reference [3] see Figures 3b, e, f. It is also clear that there remains some roughness at the bottom of the hole (typically 2 or $3 \mathrm{~nm}$ ) due probably to some pollutant.

\section{Experimental set-ups}

\subsection{Apertureless configuration}

In this $\mathrm{SNOM} / \mathrm{AFM}$ configuration the probe is an apertureless metallic tip made from a tungsten wire etched by electrochemical erosion. The typical radius of curvature of the conical tip end is smaller than $50 \mathrm{~nm}$, sometimes less than $10 \mathrm{~nm}$ (measured by scanning electron microscopy SEM) as shown in Figure 2.

The tip is 90 -deg. bent at 1 or $2 \mathrm{~mm}$ from its extremity. The "L" shaped tip is then stuck onto a piezoelectric transducer and thus it oscillates periodically above the sample, and perpendicularly to the sample surface, as in AFM tapping-mode technique. The frequency of vibration is close to the resonance frequency (about $5 \mathrm{kHz}$ depending on the length of the "L" shaped tip). The vibration amplitude is chosen to be close to $50 \mathrm{~nm}$, and it is optically monitored.

A feedback system keeps the amplitude of vibration constant while the sample is scanned by the tip, giving

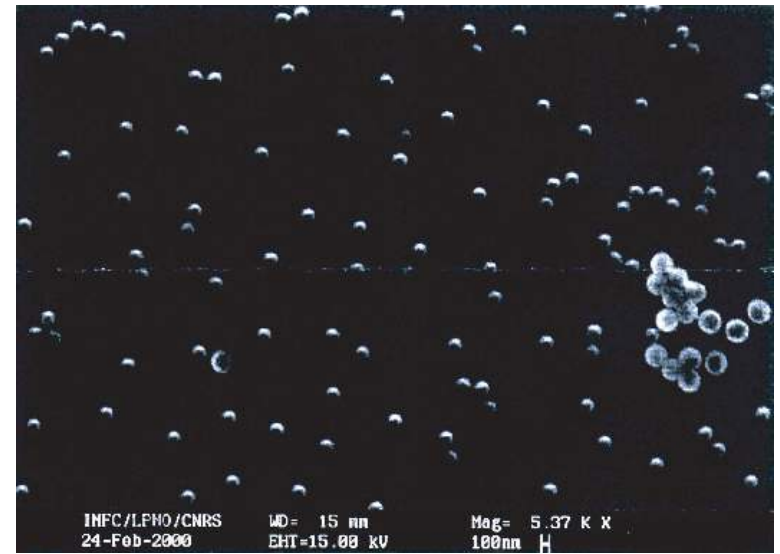

Fig. 1. SEM image of a chromium layer with random distribution of holes and a group of remaining spheres at right hand.

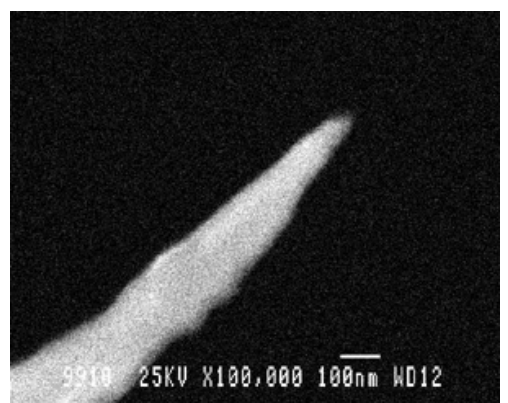

Fig. 2. SEM image of a tungsten tip.

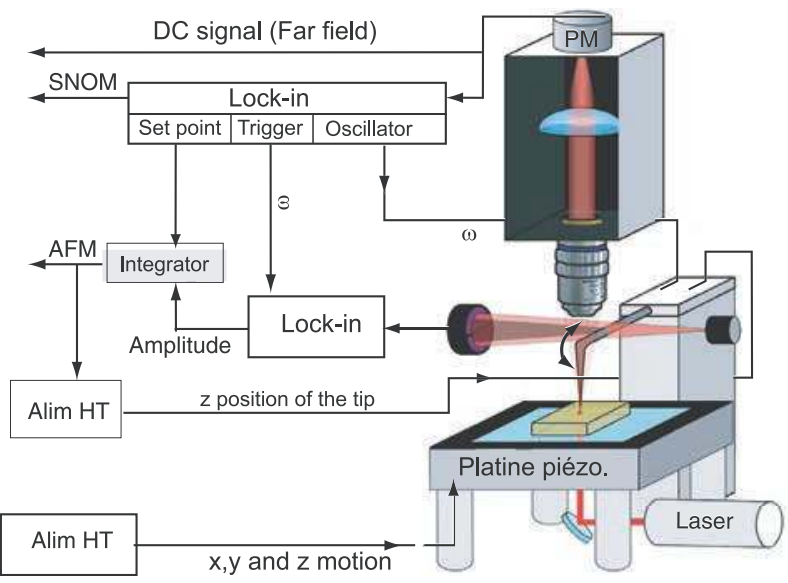

Fig. 3. Experimental apertureless SNOM set-up.

a topographical image of the sample surface. The scan is ensured by a piezoelectric translator.

The optical part is composed of a conventional microscope (Olympus BH). Various illumination modes are possible we briefly describe the transmission mode used in the experiment (Fig. 3).

A laser diode source beam (or a $\mathrm{Ti} /$ sapphire laser beam) is directed towards a first microscope objective (20X, $f=10, N A=0.4$ ), which focuses the light on the sample surface. The polarisation of the incident light is controlled externally using a polariser. The light collection ensured by a second microscope objective (for instance 
$50 X, f=6.9, N A=0.6)$ placed just above the sample and the tip, is axially symmetrical. The tip periodically scatters the near-field at the sample surface and radiates it in the far-field. This near-field contribution is recorded via a photomultiplier and a lock-in amplifier. The optical image resolution is relevant to the tip apex dimension [14].

\subsection{Isotropic STOM configuration}

The Isotropic Incoherent Scanning Tunneling Microscope (I2STOM) is a new microscope based on a particular illumination system. A quasi-polychromatic, incoherent and depolarised source generated by stimulated Raman scattering within an optical fibre is used [15]. The refraction through an axicon prism ensures an isotropic lighting of the sample in total internal reflection mode. The evanescent field is detected by the use of a stand-alone microscope head whose local probe is a nanometric tapered optical fibre working in collection mode. The tip sample distance is kept constant by means of an electronic shearforce feedback loop. The tip glued onto a tuning fork scans the sample and the near-field signal is amplified through a photomultiplier and a lock-in system. Topography and optical images simultaneously recorded are strongly correlated and optical artefacts are very low.

\subsection{Comparison of both set-ups}

Both set-ups appear quite different but they share some common properties. In both cases the sample illumination comes through the substrate and the beam shape is globally conical and axially symmetric. The near-field optical signal is collected above the hole by a feedback controlled probe. These experimental similarities will be useful for choosing the theoretical formalism used to fit the experimental results.

\section{SNOM experimental results}

Typical results obtained with the two described set-ups are shown. In each case topography and SNOM signals are recorded simultaneously for each sample.

\subsection{Apertureless configuration}

In the optical image (SNOM) of Figure 4 two lobes with opposite contrast are observed on each hole and an amplitude change occurs along the polarisation direction $(y)$.

To clarify the polarisation behaviour of the SNOM signal we have introduced in the detection channel an analyser oriented at right angle to the incident polarisation. With the tip far from the surface the detected signal is greatly reduced and we realise a detection almost in a dark field configuration. In such a configuration only the SNOM signal due to the diffusion by the tip is detected with a decrease of the signal-to-noise ratio compared to
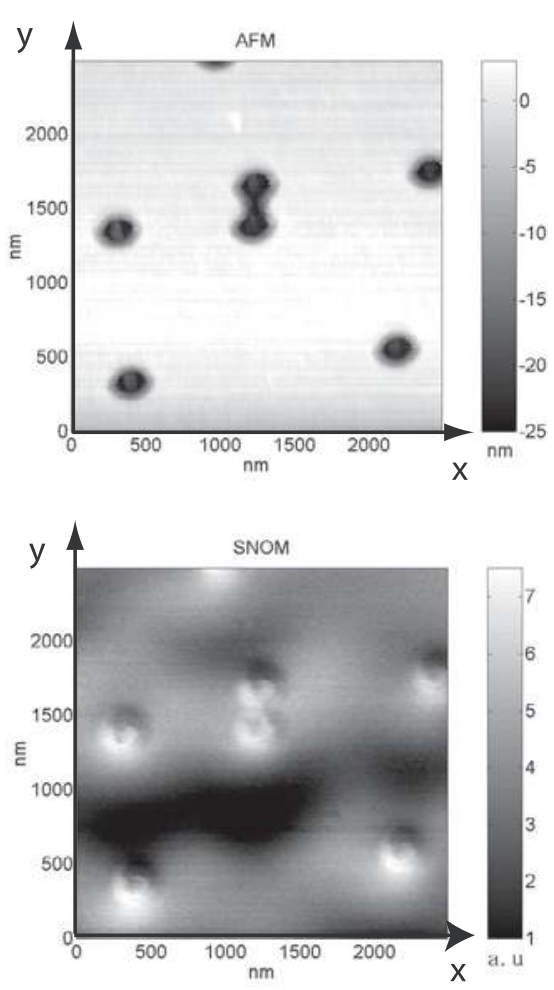

Fig. 4. AFM (top) and SNOM (bottom) images of the holes, at $\lambda=780 \mathrm{~nm}$, in the apertureless configuration. The direction of polarisation is along the vertical direction ( $y$ direction).

the one obtained without analyser. The small aperture of the objective used to focuss the incident beam on the sample prevents the longitudinal component of the polarization (along the tip axis) to become large and creating strong field enhancement on the apex of the tip [16]. The SNOM signals for two orthogonal polarisations are shown in Figure 5. The two lobes are oriented along the direction of the polariser (incident polarisation) with $x-z$ or $y-z$ mirror symmetry. They follow the polarisation after a $90-\mathrm{deg}$. angle rotation of both the polariser and the analyser.

\subsection{Isotropic SNOM results}

The test sample is isotropically illuminated in total internal reflection mode with an incoherent unpolarised polychromatic Raman source as explained above. Topography and optical images simultaneously recorded in near-field are shown in Figures 6 and 7.

Various hole distributions are observed. For example, the doublet allows us to give an estimation of the optical resolution using a separation criterion (Fig. 6 see arrow), whereas isolated apertures will quantify the optical confinement (Fig. 7).

In Figure $7[\mathrm{AB}]$ and $[\mathrm{CD}]$ cuts show that the evanescent field is confined inside the nano-hole and centred on it. The skin thickness of chrome is about $10 \mathrm{~nm}$ at middle Raman spectrum wavelength $(\lambda=590 \mathrm{~nm})$. Thus a widening of confinement is expected versus the inside hole 
a)

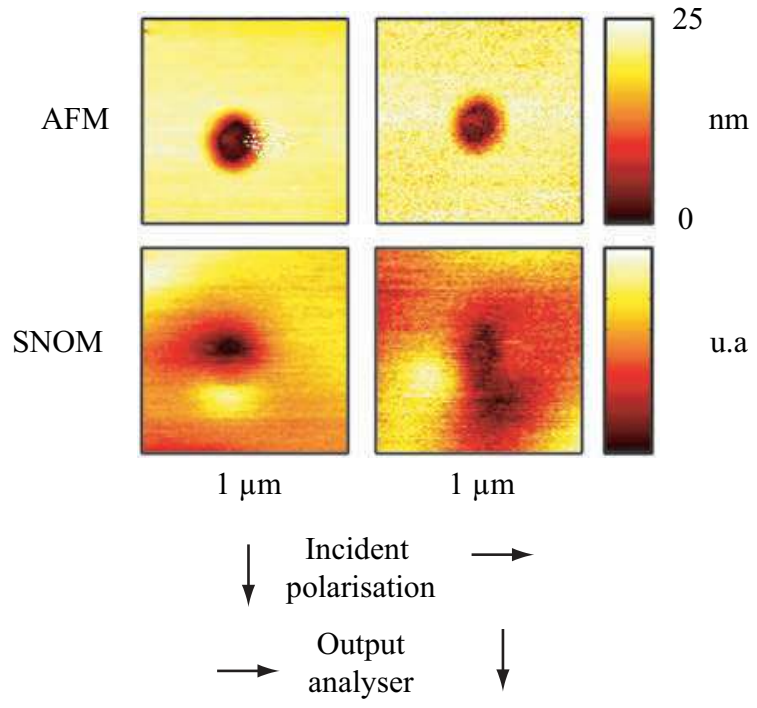

Fig. 5. AFM (top) and SNOM (bottom) images of the holes, at $\lambda=780 \mathrm{~nm}$, in the apertureless configuration. (a) Incident polarisation vertical and output polarisation horizontal (left) (b) both polarisations rotated by $90-\mathrm{deg}$. angle (right).

diameter. The estimated diameter at half-height of the topography image is $230 \mathrm{~nm}$ (see [AB] cut in Fig. 7) and the same cutting on the optical image with the confinement effect gives an estimated diameter of $270 \mathrm{~nm}$ (see [CD] cut in Fig. 7).

\section{The formalism of Grober's group}

A theoretical formalism giving the optical near-field generated by an aperture is given in reference [17]. The aperture is assumed to lie in the plane $z=0$ and diffracts an incident plane wave normal to the aperture from the half-plane $z<0$ see Figure 8 . The electric field of the incident plane wave, $\mathbf{E}=\hat{x} \exp \left[i\left(k_{0} z-\omega t\right)\right]$, is linearly polarized with the electric field oriented along the $x$ axis and of unit amplitude while $k_{0}^{2}=(\omega / c)^{2}$. The expression of the diffracted fields in the plane of the aperture is based on the analytical expressions proposed by Bethe [18] and corrected by Bouwkamp [19] for the electromagnetic field diffracted by a small aperture in a metallic plane. The techniques of Fourier optics (i.e. modal analysis) are used to describe the electromagnetic field detected by a nearfield optical probe.

Attempts to use Fourier optics to provide a quantitative understanding of this problem were initiated by $\mathrm{D}$. Van Labeke et al. [20], who used plane-wave solutions to the wave equation (in Cartesian coordinates) as the basis for a modal analysis. Also the behaviour of the Poynting vector near a small aperture was investigated numerically by Y. Leviatan [21] and T. Nakano and S. Kawata [22]. The strength of the Grober et al. analysis [17] is that: (a) the vector nature of the electromagnetic field is preserved, (b) an analytical expression for the Fourier transform of the field is provided, (c) the electromagnetic field
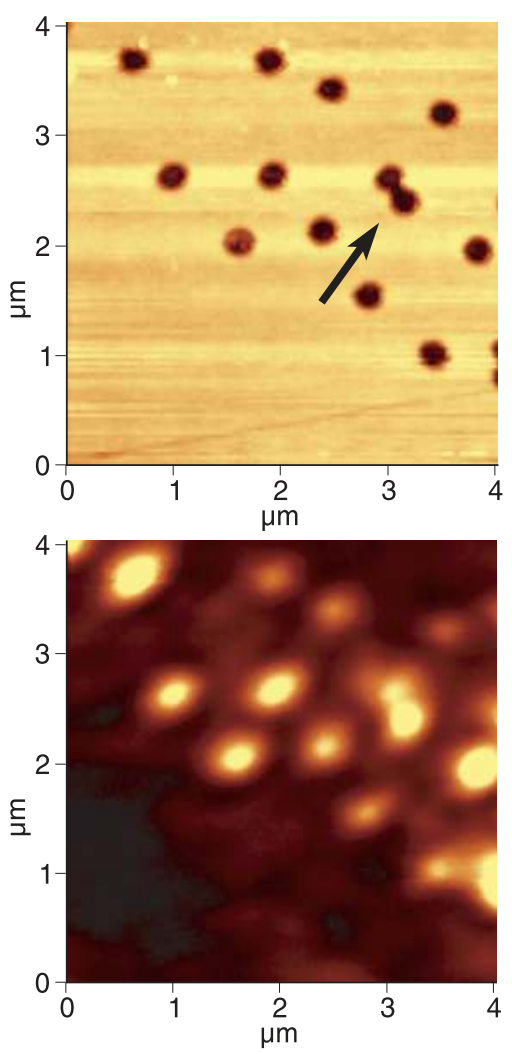

Fig. 6. Shear-force image (top) and optical image (bottom) of a distribution of holes in a chromium layer from of a typical area observed in near-field with an isotropic STOM, (scanned area $\left.4.02 \times 4.02 \mu \mathrm{m}^{2}\right)$.

can be determined by evaluating one-dimensional integrals, and (d) both the divergent behaviour of the nearfield and the dipolar nature of the far-field are recovered. Their judicious use of cylindrical coordinate system adapted to the geometry of the aperture enables to reduce the modal expansion from a two-dimensional integral to a one-dimensional integral.

If the complex time factor, $\exp (-i \omega t)$, is omitted since it plays no role in the derivation the transverse magnetic (TM) and transverse electric (TE) expansion coefficients, $f_{T M}(k)$ and $f_{T E}(k)$, respectively, are defined as

$$
\begin{aligned}
\mathbf{E}(\rho, \theta, z)= & \int_{0}^{\infty} d k\left[f_{T M}(k) \mathbf{E}_{T M}(k, \rho, \theta, z)\right. \\
& \left.+f_{T E}(k) \mathbf{E}_{T E}(k, \rho, \theta, z)\right], \\
\mathbf{B}(\rho, \theta, z)= & \int_{0}^{\infty} d k\left[f_{T M}(k) \mathbf{B}_{T M}(k, \rho, \theta, z)\right. \\
& \left.+f_{T E}(k) \mathbf{B}_{T E}(k, \rho, \theta, z)\right],
\end{aligned}
$$

where $k$ is the lateral spatial frequency, i.e. $k_{\rho}$.

By an inverse transformation of these equations, Grober et al. [17] are able to determine $f_{T M}(k)$ and $f_{T E}(k)$ from the fields in the plane of the aperture, $\mathbf{E}(z=$ $0)$ and $\mathbf{B}(z=0)$. The azimuthal symmetry of Bouwkamp's solution enables a dramatic simplification of the modal 

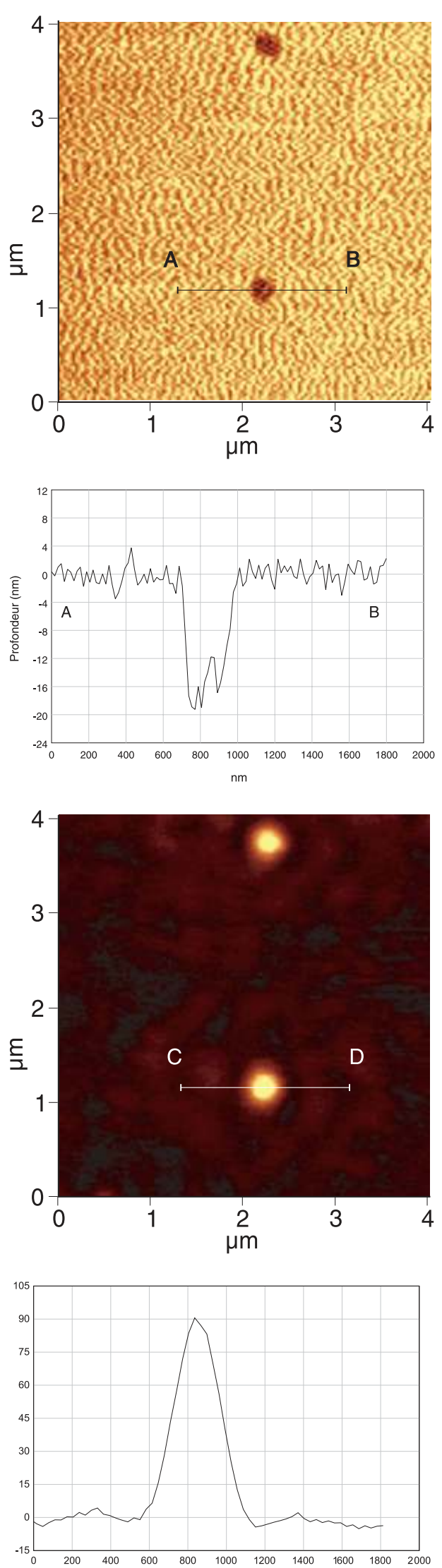

Fig. 7. Near-field confinement inside the holes. Shear-force and optical images (top and middle), corresponding profiles (below) respectively. Scanned area $4.02 \times 4.02 \mu \mathrm{m}^{2}$. expansion by requiring only the $n= \pm 1$ terms. Let us denote by $\kappa$ the longitudinal spatial frequency i.e. $k_{z}$. When $k>k_{0} \quad \kappa \rightarrow i \kappa$ and the modes become evanescent. The components of the electric field are given as follows:

$$
\begin{aligned}
E_{\rho}= & \int_{0}^{k_{0}}\left[i \kappa \cos \theta \frac{\partial J_{1}(k \rho)}{\partial(k \rho)} f_{T M}^{1}\right] \exp (i \kappa z) d k \\
& +\int_{k_{0}}^{\infty}\left[i \kappa \cos \theta \frac{\partial J_{1}(k \rho)}{\partial(k \rho)} f_{T M}^{2}\right] \exp (i \kappa z) d k \\
& +\int_{0}^{\infty}\left[-k_{0} \cos \theta \frac{J_{1}(k \rho)}{k \rho} f_{T E}\right] \exp (i \kappa z) d k \\
E_{\theta}= & \int_{0}^{k_{0}}\left[-i \kappa \sin \theta \frac{J_{1}(k \rho)}{k \rho} f_{T M}^{1}\right] \exp (i \kappa z) d k \\
& +\int_{k_{0}}^{\infty}\left[-i \kappa \sin \theta \frac{J_{1}(k \rho)}{k \rho} f_{T M}^{2}\right] \exp (i \kappa z) d k \\
& +\int_{0}^{\infty}\left[k_{0} \sin \theta \frac{\partial J_{1}(k \rho)}{\partial(k \rho)} f_{T E}\right] \exp (i \kappa z) d k \\
E_{z}= & \int_{0}^{k_{0}}\left[k \cos \theta J_{1}(k \rho) f_{T M}^{1}\right] \exp (i \kappa z) d k \\
& +\int_{k_{0}}^{\infty}\left[k \cos \theta J_{1}(k \rho) f_{T M}^{2}\right] \exp (i \kappa z) d k .
\end{aligned}
$$

Similarly the components of the magnetic field are given, thus:

$$
\begin{aligned}
B_{\rho}= & \int_{0}^{k_{0}}\left[i k_{0} \sin \theta \frac{J_{1}(k \rho)}{k \rho} f_{T M}^{1}\right] \exp (i \kappa z) d k \\
& +\int_{k_{0}}^{\infty}\left[i k_{0} \sin \theta \frac{J_{1}(k \rho)}{k \rho} f_{T M}^{2}\right] \exp (i \kappa z) d k \\
& +\int_{0}^{\infty}\left[-\kappa \sin \theta \frac{\partial J_{1}(k \rho)}{\partial(k \rho)} f_{T E}\right] \exp (i \kappa z) d k \\
B_{\theta}= & \int_{0}^{k_{0}}\left[i k_{0} \cos \theta \frac{\partial J_{1}(k \rho)}{\partial(k \rho)} f_{T M}^{1}\right] \exp (i \kappa z) d k \\
& +\int_{k_{0}}^{\infty}\left[i k_{0} \cos \theta \frac{\partial J_{1}(k \rho)}{\partial(k \rho)} f_{T M}^{2}\right] \exp (i \kappa z) d k \\
& +\int_{0}^{\infty}\left[-\kappa \cos \theta \frac{J_{1}(k \rho)}{k \rho} f_{T E}\right] \exp (i \kappa z) d k \\
B_{z}= & \int_{0}^{\infty}\left[i k \sin \theta J_{1}(k \rho) f_{T E}\right] \exp (i \kappa z) d k
\end{aligned}
$$

where $J_{1}(k \rho)$ is a Bessel function of the first kind.

The relevant optical modes for the region $z>0$ having been determined, the remaining analysis of Grober et al. [17] is to determine analytical forms for $f_{T M}(k)$ and $f_{T E}(k)$ while maintaining a physically meaningful result. The expressions obtained are given below

$$
f_{T M}^{1}\left(k<k_{0}\right)=-\frac{4 k_{0} a^{3}}{3 \pi} \exp \left(-i k_{0} a\right) \frac{k}{\left(k_{0}^{2}-k^{2}\right)^{1 / 2}}
$$




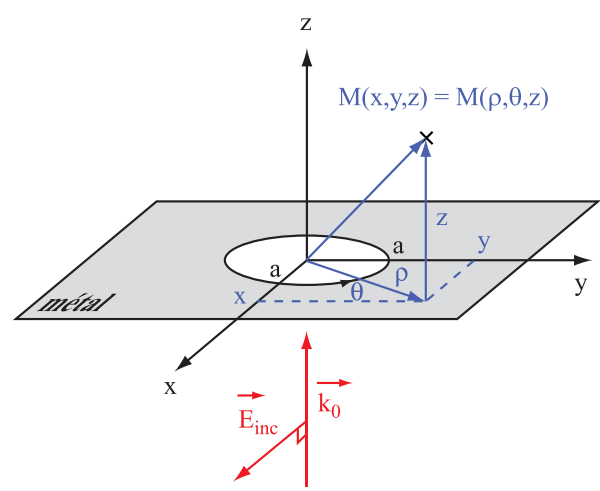

Fig. 8. Scheme of the coordinate system used in the calculation.

$$
\begin{array}{r}
f_{T M}^{2}\left(k \geq k_{0}\right)=\frac{4 i k_{0} a^{3}}{3 \pi} \exp \left(-i k_{0} a\right) \\
{\left[-1+\frac{k}{\left(k^{2}-k_{0}^{2}\right)^{1 / 2}}+\frac{\sin (k a)}{k a}\right]} \\
f_{T E}(k)=\frac{4 i a^{2}}{\pi} \exp \left(-i k_{0} a\right) \jmath_{1}(k a)
\end{array}
$$

where $\jmath_{1}(k a)$ is a spherical Bessel function.

Putting back these expressions in equations $(1,2)$, Grober et al. [17] were able to calculate the diffracted field at any point in the half-plane $z>0$. The time averaged electric field intensity follows Bouwkamp's solution in the near-field and it smoothly evolves far from the aperture to the field intensity of a radiating magnetic dipole oriented along the $y$ axis. We have used the analytical expressions above to calculate the near-field components of the electromagnetic fields and the Poynting vector in the transmission mode configuration. We first calculated numerically the values of $E_{\rho}, E_{\theta}$ and $E_{z}$ using the one dimensional equations for any value of $z>0$ and then obtained the numerical values of the $E_{x}, E_{y}$ and $E_{z}$ components.

In Figure 9 we illustrate the results obtained for the electric field components $E_{x}, E_{y}$ and $E_{z}$ for $z>0$ for an incident wavelength $\lambda=780 \mathrm{~nm}$. The amplitude of the fields in the $x y$ plane at an altitude $z=6 \mathrm{~nm}$ are shown in Figure 9 (top) and their corresponding phase is shown in Figure 9 (bottom).

In Figure 10 we show the intensity of the Poynting vector defined as

$$
\text { Poynt }=\overline{P_{x}^{2}+P_{y}^{2}+P_{z}^{2}}
$$

where $P_{x}, P_{y}$ and $P_{z}$ are the Cartesian coordinates of the vector calculated at $\lambda=590 \mathrm{~nm}$.

\section{Comparison between experiments and calculations}

\subsection{Apertureless tip}

The calculated amplitude and phase signals shown in Figure 9 for the wavelength $\lambda=780 \mathrm{~nm}$ depend on the inci-

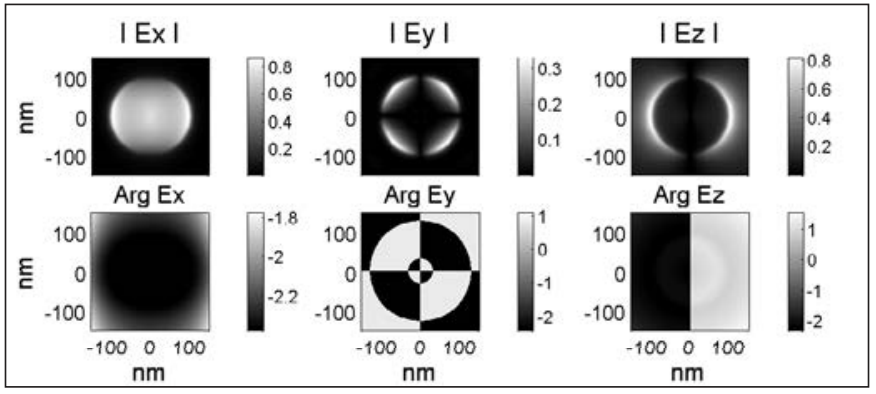

Incident polarisation

Fig. 9. Calculated electric field components at $z=6 \mathrm{~nm}$ : amplitude (top), phase (bottom).

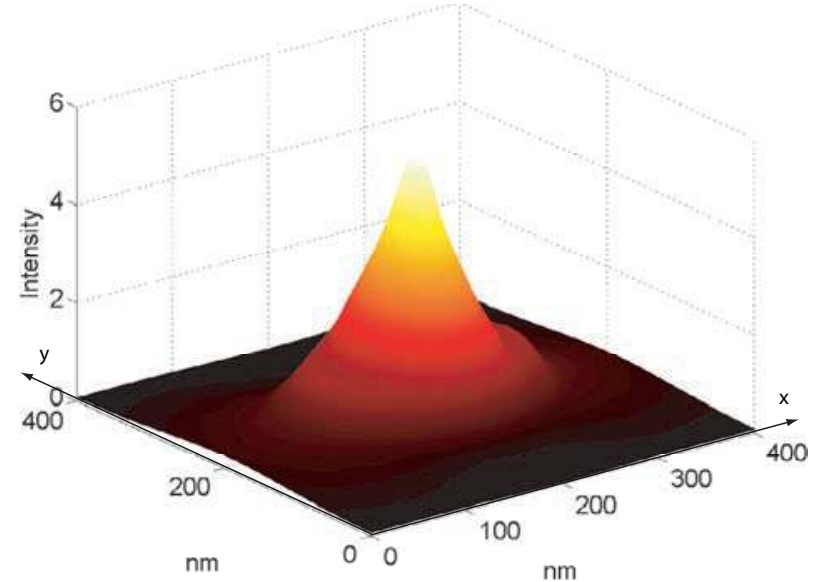

Fig. 10. Calculated Poynting vector at $z=6 \mathrm{~nm}$ and $\lambda=$ $590 \mathrm{~nm}$.

dent polarization which, in the figure, is in the horizontal direction $x$. In the apertureless configuration the SNOM signal can be seen as the result of the interference of two fields, the incident field (say $E_{r e f}$ ) and the field diffused by the tip (say $E_{\text {dif }}$ ), which is modulated at the frequency $\omega$ of the tip. $E_{r e f}$ is mainly linearly polarised and the tip converts the near-field into a radiating field whose polarisation admits a non-zero projection along the axis of polarisation of $E_{r e f}$. The photomultiplier detects the square of the modulus of the resultant field $E_{t o t}=E_{\text {ref }}+E_{\text {dif }}$ and generates a current, which can be formally written:

$$
\begin{aligned}
I_{t o t} \sim\left|E_{t o t}\right|^{2} & =\left|E_{\text {dif }}+E_{\text {ref }}\right|^{2} \\
& =\left|E_{\text {dif }}\right|^{2}+\left|E_{\text {ref }}\right|^{2}+2\left|E_{\text {dif }}\right|\left|E_{\text {ref }}\right| \cos \varphi
\end{aligned}
$$

where $\varphi$ is the relative phase of the two interfering fields. This signal enters the lock-in detector whose reference is the signal driving the modulation of the tip at the frequency $\omega$. As $\left|E_{\text {ref }}\right|^{2}$ is not modulated and $\left|E_{\text {dif }}\right|^{2}$ is modulated at twice the frequency $(2 \omega)$, the lock-in output at the frequency $\omega$ delivers the term $2\left|E_{\text {dif }}\right|\left|E_{\text {ref }}\right| \cos \varphi$, which contains the information on the phase $\varphi$ of the nearfield signal $[23,24]$.

The experimental SNOM signal detected by the lockin at the frequency of the tip can be seen in a first approximation as the difference between the signals collected 


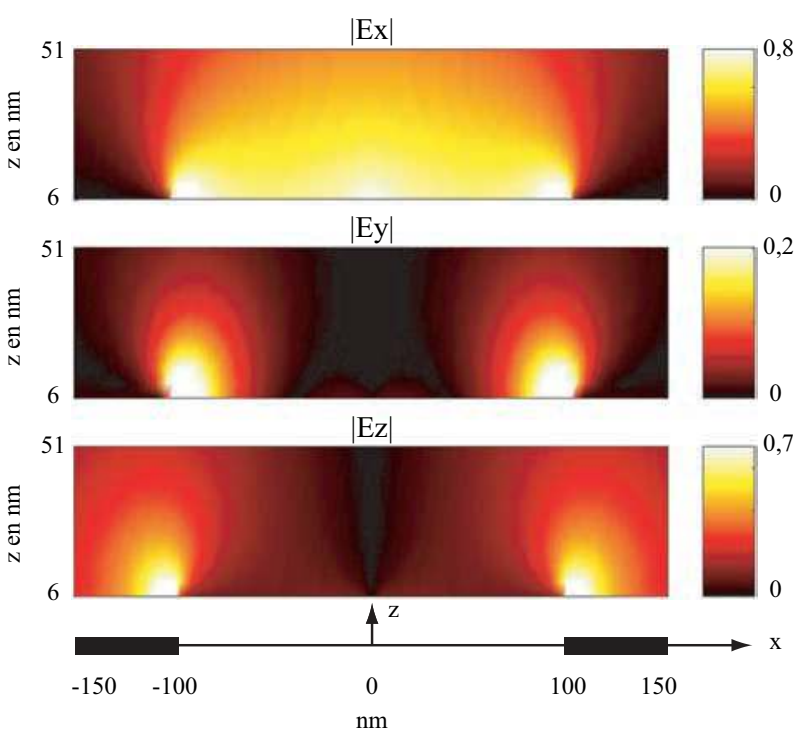

Fig. 11. Profiles of the amplitude of the electric field as a function of $z$ for $\lambda=780 \mathrm{~nm}$ : (a) $E_{x}$ (top), (b) $E_{y}$ (middle) rotated by $45 \mathrm{deg}$. with respect to the polarisation direction, (c) $E_{z}$ (bottom).

very close to the plane and well above it. To simulate the detected signal we have calculated the complex amplitudes $E_{x}, E_{y}$ and $E_{z}$ for $z=6 \mathrm{~nm}(z \simeq \lambda / 130)$ and for $z=51 \mathrm{~nm}(z \simeq \lambda / 15)$. The results are very similar to those of Figure 9 except that the fields are less localized and their amplitude is reduced. The $E_{x}$ component is almost flat all over the surface of the hole. The $E_{y}$ component presents a quadrangular distribution in both the amplitude and the phase, its amplitude maxima occurring inside the hole. The $E_{z}$ component is dominant with a clear phase variation. The behaviour of the amplitude of the field's components is illustrated in Figure 11 as a function of the altitude $z$. The profiles are taken along the incident polarisation direction for the $E_{x}$ and $E_{z}$ components. Because the $E_{y}$ component is zero along this direction the $E_{y}$ profile is shown along a line $y=x$ which corresponds to a rotation by a $45 \mathrm{deg}$. angle to the direction of polarisation. It is clear, whatever the component, that the field is highly localised in the vicinity of the hole.

Obviously the $E_{z}$ component presents the two lobes experimentally observed in Figure 5. Moreover, the calculated opposite phase change is revealed by an opposite contrast in the experimental SNOM images. This is in agreement with recent experiments [25] done using an optical phase control. The calculated and the observed signals present a mirror symmetry along the $x-z$ plane and the agreement between the calculations and the transmission experiments is satisfactory.

\subsection{Isotropic SNOM}

It has been shown [26] that a small aperture tip detects both the electric and the magnetic fields. Whether it detects the Poynting vector or the sum of the squares of the $E_{x}$ and $E_{y}$ components is not clear. Within the limitations

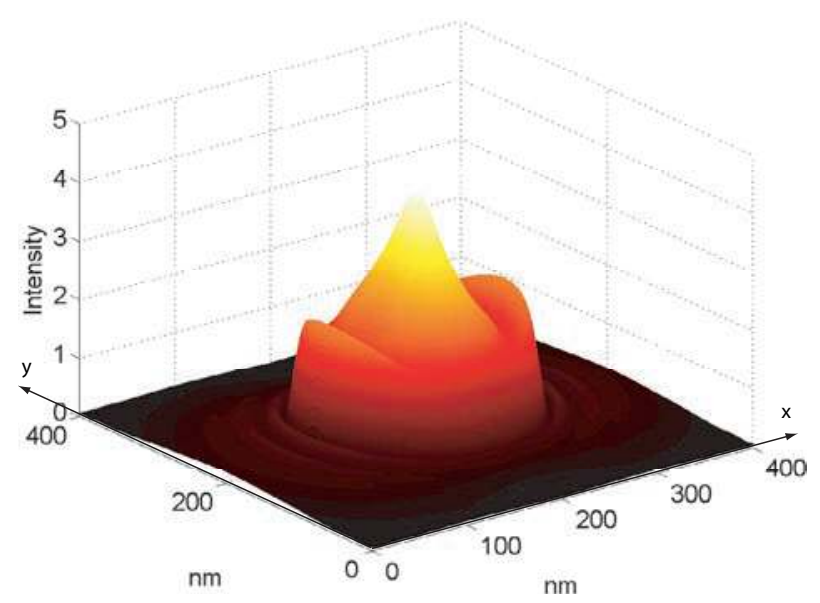

Fig. 12. Calculated $E_{x}^{2}+E_{y}^{2}$ at $z=6 \mathrm{~nm}$ and $\lambda=590 \mathrm{~nm}$.

of our crude model we have calculated both quantities in order to compare them with the experimental results.

The Poynting vector shown in Figure 10 is calculated at $\lambda=590 \mathrm{~nm}$ which is the central wavelength of the Raman spectra. The $E_{x}^{2}+E_{y}^{2}=E_{x y}^{2}$ quantity is depicted at the same wavelength in Figure 12. In both figures the polarization of the incident electric field is directed along $x$.

In the experiment done with the isotropic source the incident polarization of the electric field is also isotropic. The averaged resultant Poynting vector and $E_{x y}^{2}$ can be approximated by a weighted linear combination of the vectors or of the intensity calculated for two orthogonal polarizations. Because the source is also polychromatic, comparison with experiment requires a calculation at different wavelengths and the weighting by the incident intensity of the source components. The Raman source used in the experiment is composed of many emission lines, which span the range $532 \mathrm{~nm}$ to $680 \mathrm{~nm}$ and have quite different integrated intensities. We have made the same calculation of the Poynting vector and $E_{x y}^{2}$ as the ones shown in Figures 10 and 12 using three different wavelengths and keeping constant the $z=6 \mathrm{~nm}$ value. The weighted calculated profiles along the diameter of the hole are shown in Figure 13. Comparison with the experimental profile (see Fig. 7) leads to a better agreement with the calculated Poynting vector.

\subsection{Remark}

Several experimental factors can affect the measurements and lead to different results. Also we note that the crude theoretical formalism of Grober et al. uses an incident plane wave normal to the sample. At off-normal angles of incidence, which is the case of the two experimental results presented here the theory appears to become numerically intractable for the moment. Better adjustment could probably be gained using the conical geometry imposed by the focusing microscope objective and recently explored by Drezet et al. [27]. 


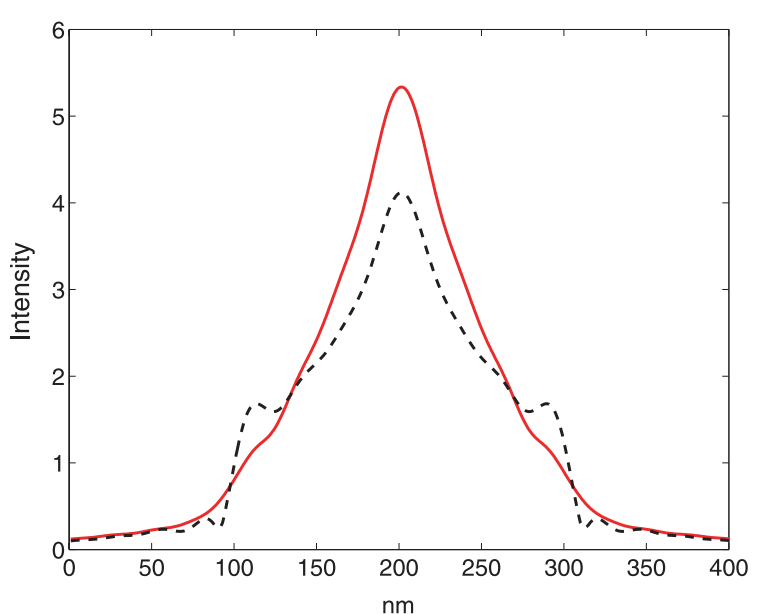

Fig. 13. Profile of the weighted calculated Poynting vector (continuous line) and $E_{x y}^{2}$ (dashed line).

\section{Discussion}

In most of the experiments done on the test object [3], the SNOM signal observed (in transmission mode) when scanning an isolated hole shows two lobes aligned with the direction of polarisation of the incident beam. Only in the case of the isotropic illumination is the SNOM signal homogeneous. In the two experiments done in transmission mode and described above, the SNOM signal resembles either the $E_{z}$ component of the near field calculated at a few nanometers above the plane $z=0$ in the apertureless configuration (see Fig. 9) or the intensity of the weighted Poynting vector which is similar to the $E_{x y}^{2}$ intensity of the electric field (see Fig. 13) in the I2STOM configuration.

Although the results shown in Figures 9 and 11 give the local field calculated without taking into account the interaction with the tip, the agreement with the experimentally detected signal is quite satisfactory. This is not surprising given the theoretical analysis of [28], which shows in particular why the SNOM transmission signals from Figure 5 follow the expected polarisation behaviour.

The existence of extremely strong field gradients at the tip apex have been demonstrated in several tip geometries [29-31]. Consideration of higher Hermite-Gaussian modes for the illumination of the tip leads also to strong field enhancements at the tip apex [9]. Whatever the shape of the probe, the calculations have been performed numerically. The total electric field generated by a plane electromagnetic wave incident on a perfectly conducting conical probe situated above a dielectric surface has been investigated [8]. The results, which are analytical, partly agree with those found in $[9,30]$. The field at a short distance $r$ from the tip apex $(k r \ll 1)$ can be written $[8,32]$ :

$$
\mathbf{E}=k(k r)^{\nu-1} \sin \beta\left[\mathbf{u}_{\mathbf{r}}+\frac{\mathbf{u}_{\theta}}{\nu} \frac{\partial}{\partial \theta}\right] a\left(\theta_{0}, \theta, \alpha\right)
$$

where $a$ is a function of the angle of incidence $\theta_{0}$, the angle of observation $\theta$, and the semi-angle of aperture $\alpha$ of the cone tip. $\mathbf{u}_{\mathbf{r}}$ and $\mathbf{u}_{\theta}$ are the unit vectors in spherical coordinates. $\nu$ is positive and smaller than 1 , and depends on $\alpha$.

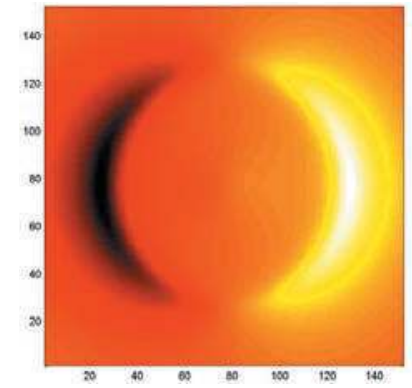

incident polarization

Fig. 14. Calculated SNOM image of a single hole assuming that the tip detects the $E_{z}$ component of the local field. (The vertical scale is the same as the one in Fig. 9). Incident polarization horizontal.

The other parameters are the wave vector $k=\omega / c$ and the angle of polarisation $\beta$ of the incident wave. While this expression of the field is asymptotic, it is not an electrostatic approximation and therefore it includes retardation effects. It is worth noticing that the field given by equation (14) is highly enhanced near the tip apex and that its spatial distribution does not depend on the illumination conditions.

In the theoretical approach of reference [28] the derivation of the signal detected in apertureless SNOM is based on the reciprocity theorem of electromagnetism. The detected field is an overlapping integral between the experimental field and a term proportional to the response function of the instrument [33], which is proportional to the derivative of the reciprocal field. To determine an approximate and practical description of this reciprocal field the authors use the expression of $\mathbf{E}$ given in equation (14). With this model their approach provides a versatile and useful tool to analyze the experimental results.

The calculations outlined above show that in apertureless SNOM configuration the tip enhances mostly the $E_{z}$ component along the axis of the cone tip. With this last assumption it is possible to compute the signal $\left|E_{z}\right| \cos \varphi$ corresponding to the interference term between the field diffused by the tip, which is sensitive to the field components oriented along $z$, and a reference wave whose modulus is 1 and its phase is zero relative to $E_{z}$. This reference wave could be the one partially transmitted by the hole. We have computed the expected SNOM signal within the model developed above and the result is shown in Figure 14.

It is clear that the calculated and experimental results are in good agreement. It would be interesting to compare these results either theoretically or experimentally with the images which could be obtained with the use of heterodyne detection $[25,34]$ or with a SNOM coupled with a Mach-Zehnder interferometer [35].

\section{Conclusion}

The study of these test objects was undertaken to compare the potentialities of the different devices currently in use in the French community of Near Field Optics. We 
have demonstrated with two transmission configurations that the topography and SNOM signals can be detected simultaneously. The vertical resolution in AFM is of the order of $1 \mathrm{~nm}$ and is limited transversely by the shape of the conical tip. Modelling SNOM observations with the Grober's formalism [17] has lead to a better understanding of the detected signals. The SNOM signals obtained with two different configurations are strikingly different but they can be described with the same model. In the I2STOM configuration the quality of the image is excellent. The recorded signals agree with the calculated intensities whereas in apertureless configuration two lobes are observed with a polarisation behaviour of the observed SNOM signal in agreement with the amplitude of the electric field.

The authors would like to thank L. Aigouy for his help in the experimental work and for providing the image of the metallic tip. They are indebted to Y. de Wilde and C. Boccara for fruitful discussions.

\section{References}

1. Proceedings of NFO-7: 7th International Conference on Near Field Optics and Related Techniques, 11-15 August 2002, University of Rochester, NY, USA

2. Le champ proche optique, Théorie et applications, edited by D. Courjon, C. Bainier (Springer-Verlag, Paris, 2001)

3. C. Bainier, C. Vannier, D. Courjon, J.C. Rivoal, S. Ducourtieux, Y. de Wilde, L. Aigouy, F. Formanek, L. Belliard, P. Siry, B. Perrin, Appl. Opt. 42, 691 (2003)

4. H.J. Lezec, A. Degiron, E. Devaux, R.A. Linke, L. MartinMoreno, F.J. Garcia-Vidal, T.W. Ebbesen, Science 297, $820(2002)$

5. D. Barchiesi, D. Van Labeke, STOM: Theoretical study of polarisation effects with two models of tip; in NearField Optics, edited by D.W. Pohl, D. Courjon, NATO ASI Series, Vol. 242, 1993, p. 179

6. J.C. Weeber, J.R. Krenn, A. Dereux, B. Lamprecht, Y Lacroute, J.P. Goudonnet, Phys. Rev. B 64, 045411 (2001)

7. A. Roberts, J. Appl. Phys. 70, 4045 (1991)

8. H. Cory, A.C. Boccara, J.C. Rivoal, A. Lahrech, Microwave Opt. Technol. Lett. 18, 120 (1998)

9. L. Novotny, E.J. Sanchez, X.S. Xie, Ultramicroscopy $\mathbf{7 1}$, $21(1998)$
10. E.J. Sanchez, L. Novotny, X.S. Xie, Phys. Rev. Lett. 82, 4014 (1999)

11. F. Formanek, Y. De Wilde, L. Aigouy, J. Appl. Phys. 93, 9548 (2003) and references therein

12. S. Grésillon, L. Aigouy, A.C. Boccara, J.C. Rivoal, X. Quelin, C. Desmaret, P. Gadenne, V.A. Shubin, A.K. Sarychev, V.M. Shalaev, Phys. Rev. Lett. 82, 4520 (1999)

13. R. Micheletto, H. Fukuda, M. Ohtsu, Langmuir 11, 44 (1995)

14. A. Lahrech, R. Bachelot, P. Gleyses, A.C. Boccara, Appl. Phys. Lett. 71, 575 (1997); S. Grésillon, S. Ducourtieux, A. Lahrech, L. Aigouy, J.C. Rivoal, A.C. Boccara, Appl. Surf. Sci. 164, 118 (2000); S. Grésillon, H. Cory, J.C. Rivoal, A.C. Boccara, J. Opt. A: Pure Appl. Opt. 1, 178 (1999)

15. C. Vannier, C. Bainier, D. Courjon, Opt. Commun. 175, $83(2000)$

16. A. Bouhelier, M. Beversluis, A. Hartschuh, L. Novotny, Phys. Rev. Lett. 90, 013903 (2003)

17. R.D. Grober, T. Rutherford, T.D. Harris, Appl. Opt. 19, 3488 (1996)

18. H.A. Bethe, Phys. Rev. 66, 163 (1944)

19. C.J. Bouwkamp, Philips Res. Rep. 5, 321 (1950)

20. D. Van Labeke, F. Baida, D. Barchiesi, D. Courjon, Opt. Commun. 114, 470 (1995)

21. Y. Leviatan, J. Appl. Phys. 60, 1577 (1986)

22. T. Nakano, S. Kawata, J. Modern Opt. 39, 645 (1992)

23. S. Ducourtieux, Ph.D. thesis, Université Paris VI, 2001

24. S. Aubert, A. Bruyant, S. Blaize, R. Bachelot, G. Lerondel, S. Hudlet, P. Royer, J. Opt. Soc. Am. B 20, 2117 (2003)

25. R. Hillenbrand, F. Keilmann, Phys. Rev. Lett. 85, 3029 (2000)

26. D. Van Labeke, D. Barchiesi, J. Opt. Soc. Am. A 10, 2193 (1993)

27. A. Drezet, J.C. Woehl, S. Huant, Europhys. Lett. 54, 736 (2001)

28. J.A. Porto, R. Carminati, J.J. Greffet, J. Appl. Phys. 88, $4845(2000)$

29. W. Denk, D.W. Pohl, J. Vac. Sci. Technol. B 9, 510 (1991)

30. O.J.F. Martin, C. Girard, Appl. Phys. Lett. 70, 705 (1997)

31. F. H'Dhili, R. Bachelot, G. Lerondel, D. Barchiesi, P. Royer, Appl. Phys. Lett. 79, 397 (2001)

32. L. Aigouy, F.X. Andreani, C. Boccara, J.C. Rivoal, J.A. Porto, R. Carminati, J.J. Greffet, R.J.C. Megy, Appl. Phys. Lett. 76, 4019 (2000)

33. J.J. Greffet, R. Carminati, Prog. Surf. Sci. 56, 133 (1997)

34. A. Nesci, R. Dändliker, H.P. Herzig, Opt. Lett. 26, 208 (2001)

35. P.L. Phillips, J.C. Knight, J.M. Pottage, G. Kakarantzas, P.S.J. Russel, Appl. Phys. Lett. 76, 541 (2000) 